\title{
Videollamadas para reducir el aislamiento social y la soledad en las personas mayores: una revisión rápida.
}

Un resumen SUPPORT de una revisión sistemática Cochrane. Julio 2020

En el contexto de la pandemia de COVID-19, muchos países han introducido restricciones de movimiento para limitar la diseminación del virus. Dichas restricciones pudieran provocar una mayor soledad y aislamiento social entre los ancianos, lo que pudiera llevar a un deterioro de su salud mental y física. La videotelefonía puede ayudar a que este grupo poblacional a mantenerse conectado durante la crisis actual al expandir sus círculos sociales o aumentar la frecuencia de contacto con conocidos.

\section{Mensajes claves:}

- Es incierto si las videollamadas comparadas con el cuidado habitual tienen algún efecto en la soledad, los síntomas depresivos o la calidad de vida (certeza de la evidencia muy baja).

- Los estudios incluidos en la revisión no reportaron datos respecto del desenlace aislamiento social.
¿Para quién es este resumen? Todo público - UPSIE, MINSAL Chile

Este resumen incluye: Hallazgos clave de la inwestigación basada en (1)

Consideraciones sobre la relerancia oe Consideraciones sebre la relerancis hvestigación para los paises de bajosimediances ingresos.

No incluido:

Reccmendaciones

Evidencia adicional no incluida en la revisión sisternatica

- Descripciones detalladas de las intervenciones o su implementación.

Este resumen se basa en la siguiente revisión sistemática

video calls for reducing social isolation and loneliness in older people: a rapid review

Qué es una revisión sistemática? Un resumen de los estudios que abordan una pregunta claramente formulada que utîzza métodos sistemáticos y explícitos para identificar, seleccionar y evaluar criticamente la investigación relevante, y para recopilar y analizar los datos de los estudios incluidos

SUPPORT fue un proyecto internecional para apoyar el uso de revisiones y estudios de politicas relevantes para informar las decisiones sobre salud materno-infantil en decisiones sobre salud materno-infantil paises de ingresos bajos y medianos, linanciado por la Comision Europea (FP6) y Institutos Canadienses de Investigacion
Salud. De este proyecto se deriva la netodologia para desarrollar estos resúmenes. 


\section{INTRODUCCIÓN}

Las restricciones de movimiento, impuestas en el contexto de las medidas para conterner la pandemia de COVID-19, puede llevar a un aumento de la soledad y aislamiento social de los grupos vulnerables de la población, y puede ser especialmente relevante para los adultos mayores. Estos dos conceptos, aunque relacionados, pueden ser definidos en forma separada. La soledad ha sido definida como "una sensación subjetiva negativa asociada con una carencia percibida de una red social amplia (soledad social) o la ausencia de una compañia social específica deseada (soledad emocional)". Asimismo el aislamiento social ha sido conceptualizado como "la perdida objetiva o escasez de contactos e interacciones con miembros de la familia, amigos o la comunidad".

Se ha observado que tanto la soledad como el aislamiento social están asociados a depresión y calidad de vida (razón por la cuál dichos desenlaces fueron incluidos en la revisión).

\section{¿Cómo se preparó este} resumen?

Después de buscar ampliamente revisiones sistemáticas que puedan ayudar a informar las decisiones sobre los sistemas de salud, hemos seleccionado las que brindan información relevante para COVID-19. Los métodos utilizados para evaluar la confiabilidad de la revisión y para emitir juicios sobre su relevancia se describen aquí:

www.supportsummaries.org/howsupport-summaries-are-prepared/

Saber lo que no se sabe es importante

Una revisión confiable podria no encontrar estudios con evidencia directa para COVID-19. Aunque eso es decepcionante, es importante saber lo que no se sabe tan bien como lo que se sabe.

La falta de evidencia no significa una falta de efectos. Significa que los efectos son inciertos. Cuando hay falta de evidencia, se debe considerar monitorear y evaluar los efectos de la intervención, si se utiliza.

Las videollamadas pueden reducir la soledad y el aislamiento social a través de la ampliación de los círculos sociales del adulto mayor o a través de un aumento en la frecuencia de los contactos con la familia, amigos u otros integrantes de las redes sociales. 


\section{Acerca de la revisión sobre la cual trata este resumen}

Objetivo de la revisión:Evaluar la efectividad de las video llamadas para reducir el aislamiento social y la soledad en adultos mayores. Además se evaluó el efecto de las video llamadas en los síntomas depresivos y la calidad de vida.

\begin{tabular}{|c|c|c|}
\hline Tipo de & Lo que buscaron los autores de la revisión & Qué encontraron los autores \\
\hline $\begin{array}{l}\text { Diseños de } \\
\text { estudios }\end{array}$ & $\begin{array}{l}\text { Estudios controlados aleatorizados (ECA) y 'cuasi-ECA' } \\
\text { (incluidos los diseños por conglomerado) }\end{array}$ & $\begin{array}{l}3 \text { estudios 'cuasi-aleatorizados' por } \\
\text { conglomerado. }\end{array}$ \\
\hline Intervenciones & $\begin{array}{l}\text { Cualquier intervención que use Internet para promover } \\
\text { una videoconferencia o una videollamada a través de } \\
\text { una computadora, teléfono inteligente o tableta, con el } \\
\text { objetivo de reducir la soledad de los adultos o el } \\
\text { aislamiento social, o ambos }\end{array}$ & Videollamadas. \\
\hline Participantes & $\begin{array}{l}\text { Las muestras de los estudios incluidos debían tener una } \\
\text { edad media de al menos } 65 \text { años. Se incluyeron estudios } \\
\text { con participantes independientemente de si } \\
\text { experimentaban o no síntomas de soledad o aislamiento } \\
\text { social al inicio del estudio. }\end{array}$ & $\begin{array}{l}\text { Todos los estudios se centraron en } \\
\text { participantes con una edad media de } 65 \\
\text { años o más. }\end{array}$ \\
\hline Ámbitos & Población general. & Hogares de ancianos en Taiwan. \\
\hline Desenlaces & $\begin{array}{l}\text { Soledad, aislamiento social, síntomas de depresión y } \\
\text { calidad de vida. }\end{array}$ & $\begin{array}{l}\text { Soledad, medida con UCLA Loneliness } \\
\text { Scale: puntajes } 20 \text { (menos solo) a } 80 \\
\text { (mas solo). } \\
\text { Síntomas de depresión, medidos con } \\
\text { Geriatric Depression Scale: puntajes } 0 \\
\text { (mejor) a } 30 \text { (peor). } \\
\text { Calidad de vida, medida con la } \\
\text { adaptación Taiwanesa del SF-36 }\end{array}$ \\
\hline \multicolumn{3}{|c|}{ Fecha de la búsqueda más reciente: 7 April 2020.} \\
\hline \multicolumn{3}{|c|}{ Limitaciones: Esta es una revisión con limitaciones metodológicas menores (evaluada con herramienta AMSTAR 2) } \\
\hline
\end{tabular}




\section{RESUMEN DE LOS HALLAZGOS}

Se identificaron tres estudios 'cuasialeatorios', que

incluyeron 201 participantes. Los estudios incluidos

compararon las intervenciones de videollamadas con la

atención de rutina en hogares de ancianos. Ninguno de estos

estudios se realizó durante la pandemia de COVID-19.

\section{Intervenciones de videoconferencia en comparación con la atención habitual para reducir la soledad}

- Las videollamadas podrían resultar en poca o ninguna diferencia en la soledad en comparación con la atención habitual a los tres meses de seguimiento (certeza de la evidencia muy baja).

- Ninguno de los estudios incluidos informó sobre el desenlace aislamiento social.

- Las videollamadas podrían resultar en poca o ninguna diferencia en los síntomas de depresión en comparación con la atención habitual a los tres meses de seguimiento (certeza de la evidencia muy baja).

- Las videollamadas podrían resultar en poca o ninguna diferencia en la calidad de vida de los pacientes en comparación con la atención habitual a los tres meses de seguimiento (certeza de la evidencia muy baja).
Sobre la certeza de la evidencia (GRADE) *

Alta: esta investigación proporciona una muy buena indicación del posible efecto. La probabilidad de que el efecto sea sustancialmente diferente $\dagger$ es baja.

Moderada: esta investigación proporciona una buena indicación del posible efecto. La probabilidad de que el efecto sea sustancialmente diferente $\dagger$ es moderada.

Baja: esta investigación proporciona alguna indicación del posible efecto. Sin embargo, la probabilidad de que sea sustancialmente diferente $\dagger$ es alta.

Muy baja: esta investigación no proporciona una indicación confiable del efecto probable. La probabilidad de que el efecto sea sustancialmente diferente $†$ es muy alta.

- Esto a veces se denomina "calidad de evidencia" o "confianza en el estimador de efecto". † Sustancialmente diferente = una diferencia lo suficientemente grande como para que pueda afectar una decisión.

Ver la última página para más información. 


\section{Intervenciones de videoconferencia en comparación con la atención habitual para reducir la soledad}

Población: Adulto mayor.

Ámbitos: Hogares de ancianos en Taiwan.

Intervención: Uso de internet para facilitar las videollamadas a través de computadoras portátiles y teléfonos inteligentes con la intención de reducir la soledad

Comparación: Cuidado estándar.

\begin{tabular}{|c|c|c|c|}
\hline Desenlace & $\begin{array}{l}\text { № estudios } \\
\text { (participante } \\
\text { s) }\end{array}$ & Impacto & $\begin{array}{l}\text { Certeza } \\
\text { de la } \\
\text { evidencia } \\
\text { (GRADE) }\end{array}$ \\
\hline Soledad * & $\begin{array}{c}3(201 \\
\text { particioantes) }\end{array}$ & $\begin{array}{l}\text { La puntuación media de soledad en los grupos de control fue de } \\
\text { 48.45. Para el grupo intervenido, a los } 3 \text { meses de seguimiento, } \\
\text { hubo una diferencia media de }-0,44(95 \%-3,28 \text { a } 2,41)\end{array}$ & $\begin{array}{c}\text { Muy baja } \\
\oplus \bigcirc \bigcirc \bigcirc\end{array}$ \\
\hline $\begin{array}{l}\text { Aislamiento } \\
\text { social }\end{array}$ & \multicolumn{2}{|c|}{ Ningún estudio informó este resultado } & -- \\
\hline $\begin{array}{l}\text { Síntomas de } \\
\text { depresión ** }\end{array}$ & $\begin{array}{c}3(201 \\
\text { participantes) }\end{array}$ & $\begin{array}{l}\text { La puntuación media de los síntomas de depresión en los grupos } \\
\text { de control fue de } 12,16 \text {. Para el grupo intervenido, a los } 3 \text { meses de } \\
\text { seguimiento, hubo una diferencia media de 0,41 (IC del 95\%:-0,90 } \\
\text { a 1,72) }\end{array}$ & $\begin{array}{c}\text { Muy baja } \\
\oplus \bigcirc \bigcirc \bigcirc\end{array}$ \\
\hline $\begin{array}{l}\text { Calidad de } \\
\text { vida *** }\end{array}$ & $\begin{array}{c}1(62 \\
\text { participantes) }\end{array}$ & $\begin{array}{l}\text { En el grupo control el puntaje promedio de calidad de vida en todos } \\
\text { los dominios fue de } 52.63 \text {. Puede haber poca o ninguna diferencia } \\
\text { entre las personas asignadas a la atención habitual y las asignadas } \\
\text { a videollamadas en puntajes de } 3 \text { meses. }\end{array}$ & $\begin{array}{c}\text { Muy baja } \\
\oplus \bigcirc 0 \bigcirc\end{array}$ \\
\hline $\begin{array}{l}{ }^{*} \text { Escala UCLA d } \\
{ }^{* *} \text { Escala de dep } \\
{ }^{* * *} \text { SF-36 (adapt }\end{array}$ & $\begin{array}{l}\text { trica, de } 0 \\
\text { nesa), de }\end{array}$ & $\begin{array}{l}\text { a más alto, peor. } \\
\text { puntos, a más alto, peor. } \\
\text { puntos, a más alto, mejor. }\end{array}$ & \\
\hline
\end{tabular}




\section{RELEVANCIA DE ESTA REVISIÓN}

\section{Aplicabilidad}

Hallazgos

Todos los estudios fueron realizados en hogares de ancianos de Taiwán
Interpretación

Las realidades de los adultos mayores no institucionalizados en Chile probablemente es muy diferente a la de los participantes en los estudios que incluyó la revisión por lo que los resultados observados podrían tener poca aplicabilidad (considerando además que muestran un nivel importante de incertidumbre respecto de los potenciales efectos de las videollamadas)

\section{Equidad}

Hallazgos

No se presentan resultados específicos para los grupos con mayores desventajas, dado que se trata sólo de población institucionalizada
Interpretación

Si bien la población institucionalizada podría ser menos autovalente, también puede ser la con más recursos financieros y/o la que posee redes familiares más activas, por lo que los resultados podrían reflejar sólo el efecto en este grupo más aventajado. Por otro lado, las videollamadas como intervención podrían ser más accesibles a los grupos más aventajados financieramente dada la necesidad de contar con un dispositivo móvil o computador con conexión a redes telefónicas o de internet. 


\section{Consideraciones económicas}

Hallazgos

Interpretación

\begin{tabular}{|l|l|}
\hline Los autores de la revisión no & No se dispone de información específica respecto de las \\
intentaron identificar aspectos & necesidades de recursos para la implementación de la \\
relacionados con el costo de la & intervención, pero esto pudiera estar limitado por los \\
intervención o el uso de & requerimientos de dispositivos a través de los cuáles se realizan \\
recursos & las videollamadas
\end{tabular}

\section{Monitoreo y evaluación}

Hallazgos

La evidencia es incierta

respecto del efecto de este tipo de intervenciones en la soledad, el aislamiento social, los síntomas depresivos o la calidad de vida
Interpretación

Es necesario realizar estudios bien diseñados

metodológicamente, con tamaños de muestra adecuados y que

incluyan un mayor rango de pacientes adultos mayores

residentes en la comunidad y con grados diversos de funcionalidad, soledad y aislamiento social. 


\section{INFORMACIÓN ADICIONAL}

\section{Literatura relacionada}

Zamir S, Hennessy $\mathrm{CH}$, Taylor $\mathrm{AH}$, et al. Video-calls to reduce loneliness and social isolation within care environments for older people: an implementation study using collaborative action research. BMC geriatrics, 2018. 18(1) 62. Available from: doi: 10.1186/s12877-018-0746-y.

Milliken MC, O'Donnell S, Gibson K, et al. Older citizens and video communications: A case study. The Journal of Community

Informatics, 2012. 8(1). Link al artículo

\section{Este resumen fue preparado por}

Equipo satellite Cochrane EPOC- Chile.

\section{Conflictos de interés}

No declarados. Para detalles, ver: link a COI equipo EPOC-Chile

\section{La revisión puede ser citada como:}

Noone C, McSharry J, Smalle M, Burns A, Dwan K, Devane D, Morrissey EC. Video calls for reducing social isolation and loneliness in older people: a rapid review. Cochrane Database of Systematic Reviews 2020, Issue 5. Art. No.: CD013632. DOI: 10.1002/14651858.CD013632. Link al documento

Sobre la certeza de la evidencia (GRADE)

La "certeza de la evidencia" es una evaluación de qué tan buena indicación proporciona la investigación del posible efecto; es decir, la probabilidad de que el efecto sea sustancialmente diferente de lo que encontró la investigación. Por "sustancialmente diferente" queremos decir una diferencia lo suficientemente grande como para que pueda afectar una decisión. Estos juicios se realizan utilizando el sistema GRADE y se proporcionan para cada resultado. Los juicios se basan en el diseño del estudio (estudios aleatorizados versus estudios observacionales), factores que reducen la certeza (riesgo de sesgo, inconsistencia, evidencia indirecta, imprecisión y sesgo de publicación) y factores que aumentan la certeza (un gran efecto, una respuesta a la dosis relación y posible confusión). Para cada resultado, la certeza de la evidencia se califica como alta, moderada, baja o muy baja

Para más información sobre GRADE: wow. supportsummaries.org/grade

\section{Este resumen puede ser citado como}

Morel M, Ortiz-Muñoz LE, Pantoja T. Resumen SUPPORT de una revisión sistemática : Videollamadas para reducir el aislamiento social y la soledad en las personas mayores: una revisión rápida. 2020. doi:10.17605/OSF.IO/A2VUH. Link al documento

\section{Palabras claves}

Evidence-informed health policy, evidence-based, systematic review, health systems research, healthcare, Public health, Video calls, older people, social isolation.

Este resumen fue elaborado con el apoyo de: Centro Evidencia UC. Pontificia Universidad Católica 